\title{
An open source tool for reliability evaluation of distribution system using Monte Carlo simulation
}

\author{
Chitumodhu Bhargava, Renuka Loka \\ Department of Electrical and Electronics Engineering, Sreenidhi Institute of Science and Technology, India
}

\begin{tabular}{l} 
Article Info \\
\hline Article history: \\
Received Sep 16, 2018 \\
Revised Nov 17, 2018 \\
Accepted Jan 28, 2019 \\
\hline
\end{tabular}

\section{Keywords:}

Communication system

Distribution system

Monte Carlo simulation

Reliability

Web services

\begin{abstract}
As the demand for electricity is shooting exponentially, reliable supply of electrical energy from generation to customer is the challenging task faced by utilities. Though generation and transmission can be maintained at hundred percent reliability, reliability of entire system cannot be assured unless focus is laid on distribution system. Most of the faults are occurring on the distribution system which is directly connected to different customers. Hence, we need to intensify the attention at distribution level. Due to deregulation, private players are evolving in distribution sector which complicates power system data exchange and information integration. To cope with this scenario, reliability evaluation of distribution system through Monte Carlo Simulation (MCS) with feature of interoperability for Bus 2 of RBTS system has been introduced and analyzed using SOAP web services. Simulation is performed by developing a web application in Java and is deployed on the Glass-fish server using JAX-WS. The intent behind this proposed model is to use the internet as the transactional tool and exposing the functionality of the program for reliability evaluation for utilities to use and providing facility of interoperability through standards refinement and integration into profiles.
\end{abstract}

Copyright @ 2019 Institute of Advanced Engineering and Science. All rights reserved.

Corresponding Author:

Renuka Loka,

Department of Electrical and Electronics Engineering,

Sreenidhi Institute of Science and Technology,

Yamnampet, Ghatkesar, Hyderabad, Telangana, India 501301.

Email: renuka.vns4evr@gmail.com, Bhargav_3417@yahoo.com

\section{INTRODUCTION}

To optimize economic operation of power system and ensure customer satisfaction, promoting maximum reliability of power system should be one of the primary goals in maintaining its overall performance standards. Interruption of power supplied to the customers impacts the system in meeting the reliability requirements. In the dynamic environment of distribution network, reliability analysis must be carried out on a periodic basis. Distribution system is the major contributor of total customer interruption duration, which calls for the need to evaluate distribution system reliability at the time of planning and expansion [1]. Researchers have introduced numerous innovative methods to efficiently calculate reliability with respect to reliability indices at hierarchical level III [2]-[7]. Importance of reliability evaluation, recent trends in power system evaluation as well as analysis methods are discussed in references [8]-[10]. The significance of optimization of investments made by utilities in order to improve reliability was researched and the outcomes of reliability analysis in terms of cost and worth were addressed using scientific methods by R. Billinton et al. [11], [12]. Deregulation of power systems and open access in distribution has encouraged researchers to focus on IT enabled applications for various challenges encountered such as automation, end to end communication, and integration of information. Service oriented architecture provides loosely coupled and flexible environment in open electricity market through which information integration suitable for the needs of utilities was developed [13], [14]. The power demand increases enormously day by 
day which is caused due to increase in size of the system. Non-government industries have joined with public sector industries to meet increasing power demand. In power system operations collecting the massive data is very complex and it is stored in different formats and distributed in a heterogeneous environment. Privatization of electricity markets require heterogeneous data to be exchanged in an unambiguous way. Hence, distributed model for reliability evaluation to attain platform independent solution is of prime importance which was described in reference [15].

Because of privatization and increased competition in global electricity markets, new distribution companies emerge which tend to operate on their chosen platforms. This demands for integration of reliability information obtained from various distribution companies. This data/information exchange cannot be done when data is obtained from different platforms using a direct approach. Hence a platform independent service-oriented architecture should be developed. Also, reliability evaluation has to be performed for these private distribution companies at regular intervals using appropriate method by using the reliability data.

The challenge in integrating reliability data from various distribution companies can be answered through the web services. A web service acts as an interface between legacy power system applications. Data can be translated into a common format (XML) that is understandable by user/client applications whenever information exchange has to take place. This eradicates the ambiguities associated in sharing information providing a loosely coupled distributed model. Another added advantage is easy accessibility over the network across various locations. As the service is masked in layers, users need not be aware of the complexities involved in reliability analysis, thus making it simple for customers/clients. Hence, this can be summarized as inter-operable, transport independent, easily accessible, user friendly and scalable model.

Recently, cluster based power systems were analyzed using web service technology in reference [16]. Time sequential simulation technique generates better picture of system reliability over analytical method which was presented in [17]. This work presents a unique solution for reliability evaluation and data sharing while computing reliability indices using Monte Carlo Simulation (MCS) method by introducing an agile technology for information interchange among different distribution companies through the development of SOAP web services [18]-[23]. Reliability evaluation using analytical approach and MCS simulation approach were described and the procedure was developed. Strategic communication model for reliability data exchange between client and service provider was presented in a stratified manner using web service architecture. This model was realized using BUS-2 of RBTS standard distribution system. Web service was tested using SOAP UI (User Interface) to transport reliability indices to the distribution utilities as SOAP messages in XML format.

\section{RESEARCH METHOD}

\subsection{Basics of Failure Mode Effect Analysis}

Reliability studies for distribution systems using analytical methods utilize each component failures and their effect on system. This is called failure mode effect analysis (FMEA) which is described in reference [1]. Out of this process a list for interruption events is created so that basic load point indices are obtained. A final list of failure events is formed to evaluate the basic load point indices. The failure in the transformer, lateral, lateral section and any main section of the feeder causes to outage of load point on feeder. The reliability indices of load point $i$ is given by (1), (2), and (3). The system indices given by (4) to (10) are obtained from load point indices. The cost/worth indices given by (11) to (13) are obtained from both. The method (FMEA) adopted for analytical calculations are as described in [1]-[5].

Mathematical formulae used for calculating the load point, system and cost indices are given below: Load point Indices

$$
\begin{aligned}
& \lambda_{\mathrm{i}}=\lambda_{\mathrm{T}}+\lambda_{\mathrm{LSi}}+\lambda_{\mathrm{MS} 1}+\lambda_{\mathrm{MS} 2}+\ldots \ldots \ldots \ldots+\lambda_{\mathrm{MSi}} \\
& \mathrm{U}_{\mathrm{i}}=\lambda_{\mathrm{T}} \mathrm{r}_{\mathrm{T}}+\lambda_{\mathrm{LSi}} \mathrm{r}_{\mathrm{LSi}}+\lambda_{\mathrm{MS} 1} \mathrm{r}_{\mathrm{MS} 1}+\lambda_{\mathrm{MS} 2} \mathrm{r}_{\mathrm{MS} 2}+\ldots \ldots \ldots+\lambda_{\mathrm{MSi}} \\
& \mathrm{r}_{\mathrm{i}}=\frac{\mathrm{U}_{\mathrm{i}}}{\lambda_{\mathrm{i}}}
\end{aligned}
$$

Where,

$\lambda_{\mathrm{T}}=$ average failure rate of a transformer

$\lambda_{\mathrm{LSi}}=$ average failure rate of lateral section $\mathrm{i}$

$\lambda_{\mathrm{MSi}}=$ average failure rate of feeder main section $\mathrm{i}$ 
$\mathrm{r}_{\mathrm{T}}=$ average repair time of a transformer

$\mathrm{r}_{\mathrm{LSi}}=$ average repair time of lateral section $\mathrm{i}$

$\mathrm{r}_{\mathrm{MSi}}=$ average repair time of feeder main section $\mathrm{i}$

System indices:

1. System average frequency index, SAIFI

$$
S A I F I=\frac{\sum_{i} \lambda_{i} N_{i}}{\sum N_{i}}
$$

2. System Average Duration index, SAIDI

$$
S A I D I=\frac{\sum_{i} U N_{i}}{\sum N_{i}}
$$

3. Customer Average Interruption Duration Index, CAIDI

$$
C A I D I=\frac{\sum_{i} U_{i} N_{i}}{\sum \lambda_{I} N_{i}}
$$

Where, $\lambda_{i}$ is the failure rate in failures/year, $N_{i}$ is the number of customers of load point $i, U_{i}$ is the annual outage time in hours, and $L_{a(i)}$ is the average load connected to load point in $\mathrm{kW}$.

Cost Worth Indices

1. Expected energy not supplied,

$$
\text { EENS }=L_{p} U_{j}
$$

Where, $L_{p}$ is the average load at load point $p$ and $U_{j}$ is the outage duration for event $j$.

2. Expected customer interruption cost,

$$
\mathrm{ECOST}=\sum_{j=1}^{N C} L_{k j} f_{j} c_{j}\left(d_{j}\right)
$$

Where $L_{k j}$ is load curtailment in megawatt, $f_{j}$ is frequency in occurrence/year, $c_{j}\left(d_{j}\right)$ is the customer damage function for contingency $j$.

3. Interrupted energy assessment rate,

$$
\mathrm{IEAR}=\frac{\mathrm{ECOST}}{\mathrm{EENS}} \text { in rupees/kilowatt-hour }
$$

\subsection{Monte Carlo Simulation Procedure}

Simulation procedure considers latent volatility index in performing the calculations thus considering the real time systems [1], [2]. The basic simulation procedure depends upon generations of random numbers for relevant probability distribution for each and every element in the system for its performance. The distribution system consists of components such as distribution transformers, feeders, sections, distributors, sectionalizing switches, breakers, and alternative supply. Exponential probability distributions are used to simulate artificial failure histories. MTTF and MTTR are converted into exponential distribution using inverse transform method for performing the simulation procedure [6], [7]. Reliability indices for different systems under certain constraints were computed in an exhaustive manner in references [8]-[10]. Quantification of reliability in terms of cost is performed using reliability worth analysis [11], [12]. MCS procedure is depicted in the following flow chart. Monte Carlo simulation procedure is shown in Figure 1.

\subsection{Test System}

Bus2 of RBTS radial distribution system is considered for estimation of reliability indices [3], [4]. The circuit breakers are assumed to be $100 \%$ reliable. The operation of the transformer is considered independent of weather conditions as they are generally housed in buildings. Hence transformers failure rate is taken as a constant value.

The 33/11 kV of a Roy Billinton Test System (RBTS) which consists of all the basic components such as circuit breakers, feeders, switches, loads as well, a tie -line switch is considered for calculations of reliability indices. The considering test system does not include the additional features such as isolation, additional protection and transferable loads. The single line diagram of Bus 2 of RBTS as shown in Figure 2. 


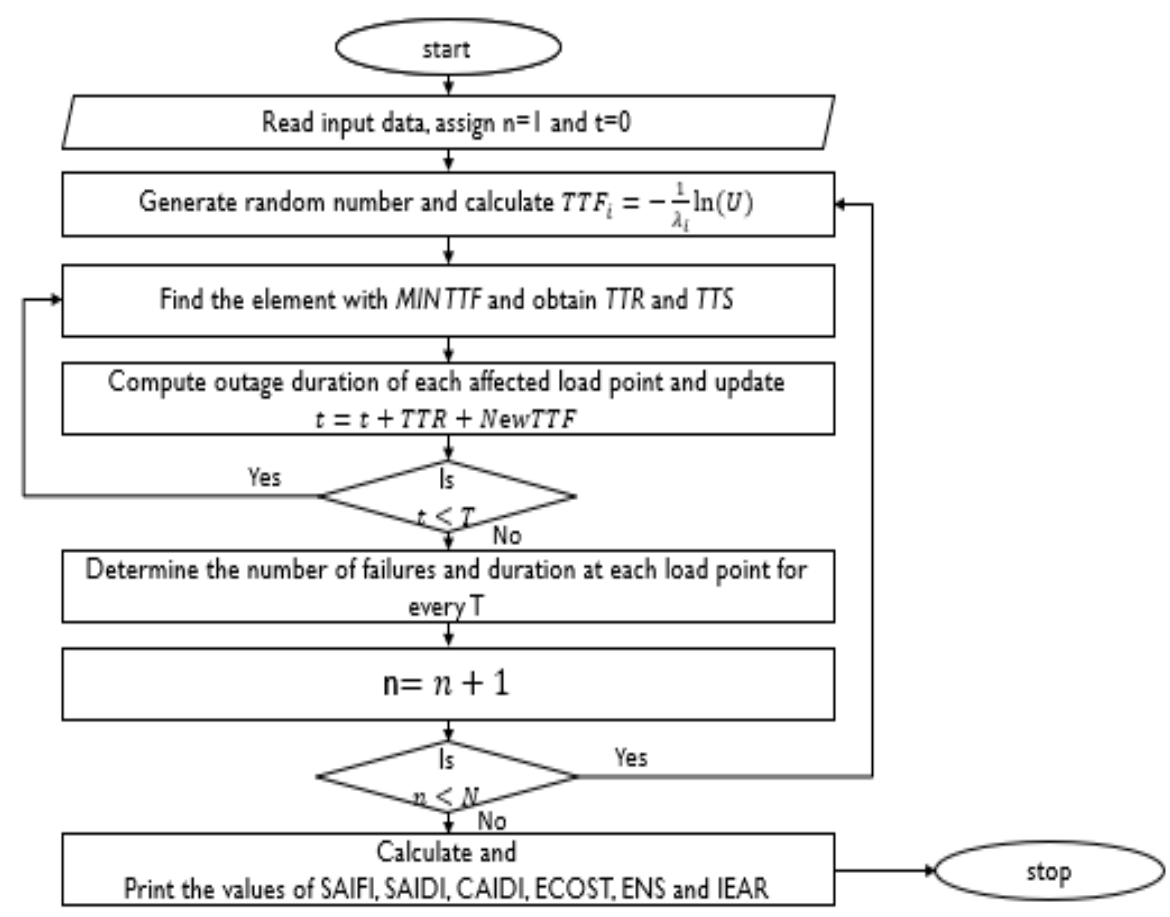

Figure 1. Monte Carlo simulation procedure

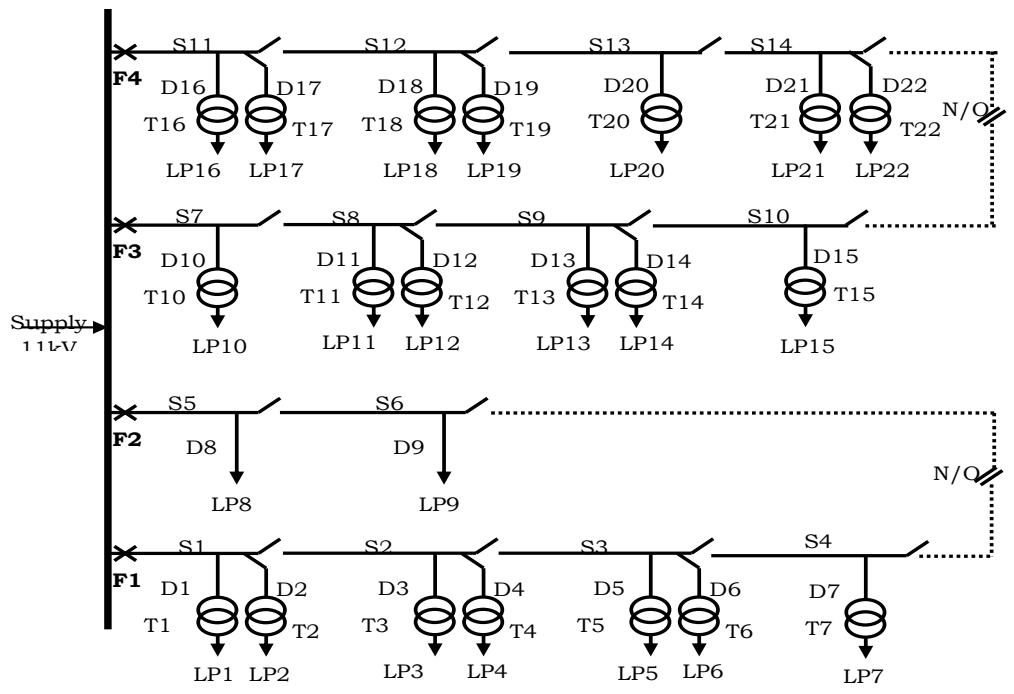

Figure 2. RBTS distribution system

\subsection{Web Service Architecture}

Open access in distribution system calls for the need of data integration and interoperable communication among different companies. An architectural model is designed to suit the current trends of distribution system [13]. Web service is an abstract representation of an interoperable framework which can be operated over the network and it has to be implemented by an agent (service provider) [14]. The present model is shown in Figure.3which depicts the interaction of utility and remote service provider through an interface. The client request is in the model of SOAP (Simple Object Access Protocol) message style which is represented in XML format and response is also generated the form of a SOAP message. The functionality of the web service is independent of operating framework of the client. The service provider entity is represented by its service as 'MCS Service', and requester entity is represented as the 'Distribution Company'. 


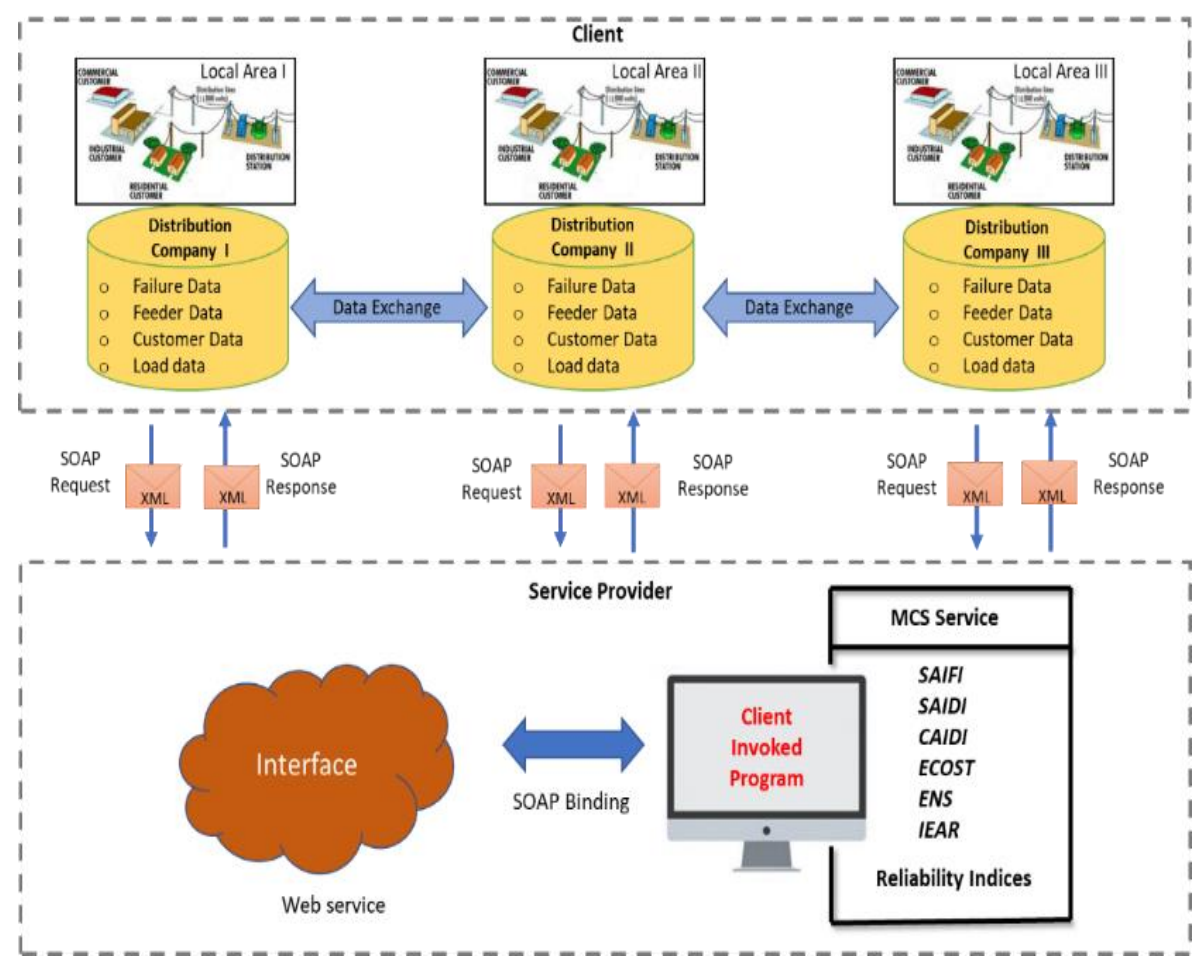

Figure 3. Web service architecture

The reliability data can be retrieved from various distribution companies which are operated at different places. Web service offers an interoperable feature for exchange of data. Along with this webservice enables communication between two power system applications irrespective of operating platform. Hence reliability service creates a distributed architecture.

Distribution Companies and service provider recognize each other through web service discovery. Each distribution company provides the reliability data involving failure data, feeder data, customer data and load data pertaining to its distribution system local area as inputs to the provider entity. These inputs are converted into SOAP messages and accesses the service provided by service provider using the URI (Unique resource Identifier) [15]. This information provided by the client initiates the program to compute the reliability indices using simulation procedure of MCS. Once the values are obtained through the client invoked program, these are sent to the distribution company using HTTP (hypertext transfer protocol) binding in XML format [16]-[18].

\subsection{Web Service Implementation}

MCS service was developed based on SOAP web Services. The reliability application in JAVA was deployed on the web server and then WSDL (Web Service Description Language)- URI (Unique Resource Identifier) was obtained. Distribution companies provide their system data as a SOAP request. Reliability indices were estimated and sent to the utility as a SOAP response by the reliability service provider.

The implementation of web service goes through the pipeline of phases like building, packaging and deploying the reliabilityservice [19]. These phases were executed using NetBeans IDE and SOAP UI software. Java packages were built using JAX-WS which stands for JAVA application program interface for SOAP web services and were deployed on the glass fish server. After deploying the service, a WSDL which includes all the service descriptions is generated. Using this information, communication is possible through SOAP message in XML format for exchange of information between utility and service provider.

\subsection{Building, Packaging and Deploying}

The data of distribution system can be versatile providing a flexible environment for any type of radial distribution feeder. As an initial step, JAVA class was written to input the data of the feeders such as number of feeders, failure rate in failures/year, length of the feeders in kilometres, number of distributors, load points, average load and peak load in kilowatt corresponding to each load point. Random number generator was used for converting the failure rate into time to failure using exponential probability distributions and simulation procedure for MCS service was built using JAVA packages. 
Later the java classes are compiled by inbuilt compiler [20]. Using simulation procedure of Monte Carlo Technique, methods for computational logic of primary indices, secondary indices and cost/worth indices were written using JAVA. As these are back end applications, user need not be aware of the computational logic. Once we compile the java Classes, using the deployment tool in net beans runtime IDE, these were deployed on the glass fish web server by starting the server in runtime.

To expose a web service, Java interface classes as well as implementation classes are required. Such interface classes are knowns as Service endpoint Interface (SEI) [21]. The extension of such an interface is as java.rmi.remote which helps to recognize itself as an interface class, where rmi stands for remote method invocation.

Service bean are the implementation classes needed for handling the incoming SOAP requests. Thus, service endpoint interface and implementation classes are generated by ATG (Art Technology Group) platform. These are necessary for implementing reliability service. Once the application was deployed on the sever, WSDL is automatically generated.

\subsection{Web Service Description Language (WSDL)}

WSDL(webservice description language) provides meta data of the service. All the definitions related to java methods and location of the host are available in WSDL [22].Using JAXRPCServlet, location details of the service such as the host and port number are imported in the target namespace. Web Service description consists of information about the URI, SOAP binding, SOAP operations, body of the SOAP messages and also the output. It is an XML document in a simple object access protocol. Thus, the semantics of the SOAP message to be invoked by the service from the distribution company side are all included in the service description. The protocol used is in the form document style for messaging and SOAP binding is defined as the HTTP (hypertext transfer protocol) binding. Input and outputs are described in the body as SOAP messages.

\subsection{Web Service Testing}

MCS service was tested using Bus 2 of RBTS (Feeder-3) which consists of six load points LP-10 to LP-15. This is the final step in developing a web service for reliability estimation. Glassfish server should be running for this purpose. Distribution company can use this SOAP UI as user interface. Otherwise, there is an inbuilt test environment available in the glass fish server for utility/distribution companies to use the reliability service according to their system configuration.

Functionality of the web service was designed such that it is irrespective of the feeder configuration such as number of sections, distributors, transformers or load points. All the inputs are in document style. Once all the necessary input message requests are sent, utility can invoke the reliability service to get all the reliability indices for the proposed feeder configuration. Thus, testing can be performed in a user-friendly environment.

In web service technology SOAP is considered as a platform independent form of communication. Data interchange between the applications over the internet using SOAP is hassle free and easy to use [18]-[23]. The messages are sent as SOAP requests in the reliability service for estimating the reliability indices. The SOAP request is sent as shown in Figure 4. Different distribution companies using different platforms can easily access this service. Encoding of these messages is done in XML (extensible mark-up language) format. Using WSDL link from the host computer, SOAP project has been tested in SOAP UI. local host implies utility or user is located on same computer.

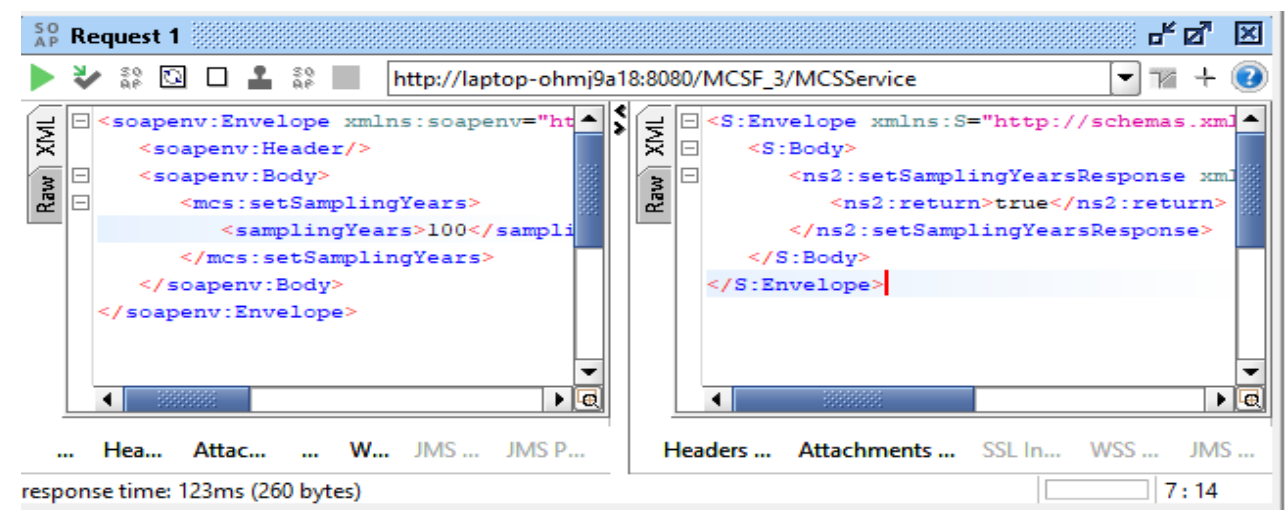

Figure 1. SOAP UI request for utility 


\section{RESULTS AND DISCUSSION}

Reliability studies are an indispensable part of power system planning and operation. At the time of power system expansion, system reliability should be assessed in order to ensure a reliable and secure supply. Whenever a severe fault occurs on the distribution system, system restoration may take place. During restoration, calculation of system reliability indices is often performed. Hence, reliability web service incorporating the feature of interoperability can be very useful for performing reliability studies at regular intervals during power system planning, operation, expansion and restoration.

Using Monte Carlo service, reliability indices are calculated for bus 2 of RBTS and transported to the utility company in order to illustrate the interoperable nature of the web service developed. Input data acquired from the utility is converted into XML format to obtain reliability indices as a SOAP response.

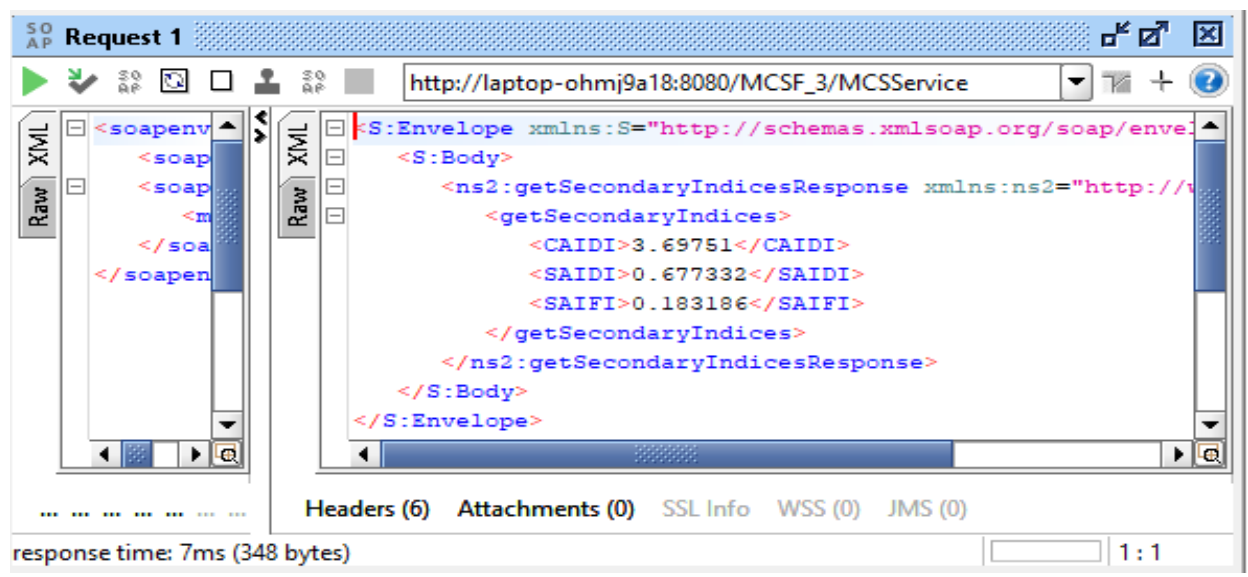

Figure 2. SOAP response for secondary indices of reliability service

Figure 5. illustrates the secondary indices response for the utility request tin XML format using SOAP-UI. Similarly, values obtained for primary indices, customer damage function and cost worth indices are transferred to the utility. It is demonstrated that interoperability between different utilities can be achieved using XML format for data transfer. It can be seen that a web browser and internet connectivity are sufficient for any distribution company for accessing the user-friendly service developed for reliability applications. It is also proven that web service requests take substantially less time for execution. The developed web service has processed the service request for Feeder-3 of bus 2 of RBTS within fifteen milliseconds. To validate the results obtained from MCS service using simulation method, rigorous calculations are performed using analytical method of reliability evaluation.

Table 1. Secondary Indices of Feeder-3

\begin{tabular}{llll}
\hline Indices & Analytical & MCS & \%error \\
\hline SAIFI(Interruptions/customer) & 0.180 & 0.183 & -1.66 \\
SAIDI (hours/customer) & 0.680 & 0.677 & 0.44 \\
CAIDI (hours/ interruption) & 3.710 & 3.697 & 0.35 \\
\hline
\end{tabular}

The comparative results of secondary indices obtained using the web service and analytical method for the considered case study are tabulated in Table 1. Error percentage calculated using analytical method and the results obtained from simulation procedure are minimal and hence accuracy of results can be identified. The maximum difference for SAIFI is -1.66 percent for Feeder-3. Similarly, for SAIDI, the maximum difference is 0.44 percent for feeder- 3 and for CAIDI, the maximum difference is 0.35 percent for Feeder-3.

Load points 10, 11 and 12 supply residential load, load points 13 and 14 supply institutional load and load point 15 supplies commercial load. Customer damage function corresponding to the type of customer was calculated and used in the evaluation of ECOST, EENS and IEAR. The comparative results for cost/worth indices for analytical and simulation methods were tabulated in Table 2. 
Table 2. Cost /Worth Indices of Feeder-3

\begin{tabular}{cccccccccc}
\hline Load & \multicolumn{2}{c}{ ECOST (kRs/year) } & \multicolumn{2}{c}{ EENS (MWh/year) } & \multicolumn{3}{c}{ IEAR (Rs/kWh) } \\
Point & Analytical & MCS & \%error & Analytical & MCS & \%error & Analytical & MCS & \%error \\
\hline & 0.49 & 0.50 & -2.04 & 0.39 & 0.40 & -2.56 & 1.24 & 1.25 & -0.80 \\
& 0.45 & 0.44 & 2.22 & 0.36 & 0.35 & 2.77 & 1.25 & 1.24 & 0.80 \\
& 0.38 & 0.37 & 2.63 & 0.30 & 0.31 & -3.33 & 1.25 & 1.24 & 0.80 \\
& 0.39 & 0.40 & -2.56 & 0.26 & 0.25 & 3.84 & 1.54 & 1.53 & 0.64 \\
& 0.38 & 0.37 & 2.63 & 0.25 & 0.24 & 4.0 & 1.55 & 1.54 & 0.64 \\
& 2.70 & 2.71 & -0.37 & 0.33 & 0.32 & 3.03 & 8.21 & 8.20 & 0.12 \\
\hline
\end{tabular}

The results in Table 2 show the cost/worth indices for each load point of Feeder-3 of Bus 2 of RBTS from both analytical and simulation using MCS service with their corresponding error percentages. The maximum deviation for these indices is: for ECOST, the maximum difference is 2.63 percent for Feeder3. Similarly, for EENs, the maximum difference is 3.84 for feeder-3 and for CAIDI, the maximum difference is 0.8 percent feeder-3. Customer Damage Function was calculated using the Web Service and used in calculating the Cost worth Indices. ECOST for load point 15 is Rs 2.71 and IEAR being 8.2 Rs/kwh contribute to most of the cost incurred with interruption for feeder-3.

\subsection{Probability Distributions of Load Point and System Indices for Feeder-3 of Bus2 of Rbts}

Failures occurring in a year is a random event and thus probability distribution provides an idea of the stochastic nature of this event by the application of MCS technique. Exponential probability distributions are used for estimating the stochastic behavior of components. Simulation time period considered in developing this web service is one year/ 8760h and the total number of years for which each of the indices are simulated to obtain the corresponding probability distributions of the indices is 100 years.

Probability distribution of failure rate for all load points over the total number of simulation years calculated using MCS web service can be represented as in Figure 8. Probability Distribution of reliability index SAIDI for all load points of Feeder-3 of Bus 2 of RBTS is shown as in Figure 9.

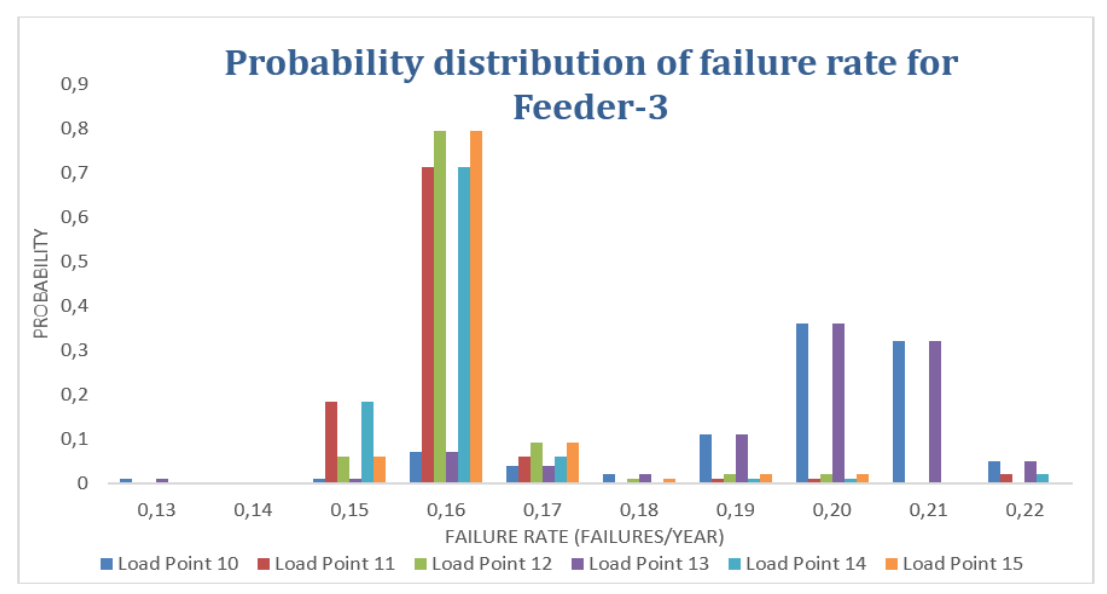

Figure 8. Probability distribution of failure rate for Feeder-3

P. Anabalagan and V. Ramachandran [15] made an attempt to design a communication prototype in a distributed environment using analytical approach for evaluation of system reliability indices. Most of the distribution companies in India and even in developing countries like western countries, causes of failure events are still manually recorded in log books, have not been properly calculated and maintained to obtain the primary indices. This has given uncertainty (number of occurrences of faults, restoration time) in input data as well as in output calculations also. In the past decades, various different techniques are used for evaluating the system reliability indices. These techniques can be roughly categorized as the simulation and analytical methods. The analytical approach gives a single average value and at the same time it does not take into consideration the uncertainty in the behavior of the system. Analytical techniques are depending on some assumptions related to the system outage records and takes more time of execution for complex systems. The simulation techniques are the most flexible due to two reasons1) It considers the random occurrence of faults 2) It gives the variability of indices. 


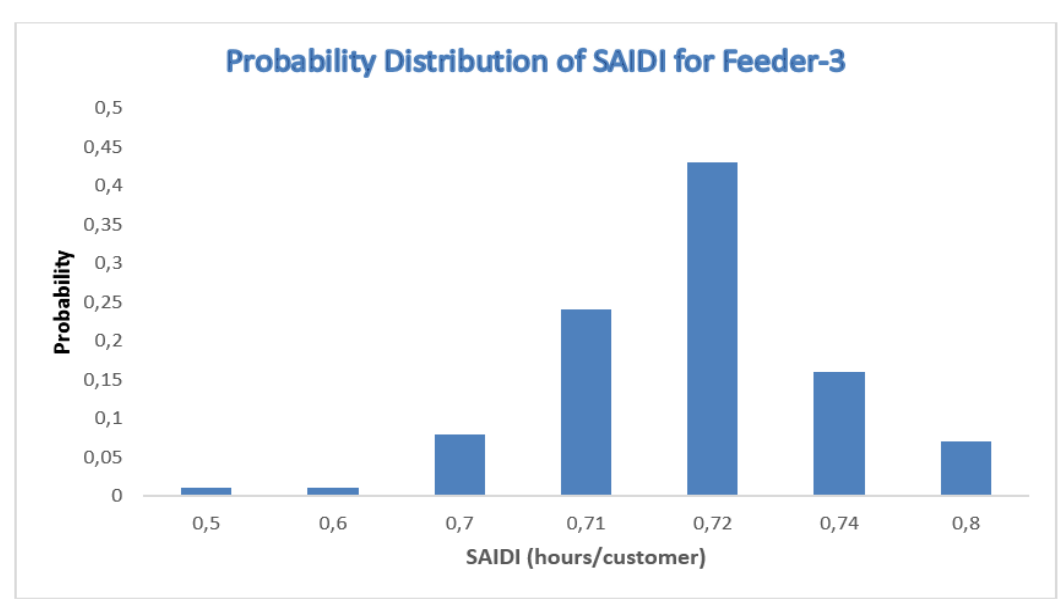

Figure 9. Probability distribution of SAIDI for Feeder-3

Reliability indices calculation is generally obtained by using uncertain variables (number of occurrences of faults, restoration time) so that reliability indices become random variables. For that a stochastic model of the operation can be used.

Monte Carlo simulation (MCS) technique deals with the uncertainty in input data and gives a complete knowledge of the probability distributions of reliability indices in addition to their average values and also gives the additional information related to variation of indices. So MCS is more practical when additional information is required and this technique solves the very difficult reliability problems using random numbers. In this simulation program for every iteration the result is stored and at the end of all iterations the sequence of results generated is transformed into frequency distribution that permits to calculate the mean and standard deviation. The simulation is performed for a period of 100 sample years. For further improving the performance and capability of MCS technique a web service technology is incorporated to calculate the reliability indices. Reliability cost/worth assessment plays an important role in the power system planning, operation and expansion and it provides an opportunity to include customer concerns in this analysis Performance and user-friendly aspects were not presented effectively in [15], [16] while using web service technology. In this paper the importance and applications of SOAP-UI was given to boost the performance of the service developed in a utility friendly environment. Soap-UI creates a user-friendly environment for customers for calculating the reliability indices and a layman can also obtain reliability information of particular feeder without having the knowledge of reliability concepts.

The above said two approaches are very useful to system planners to make utility planning and operating decisions and also useful to customers for taking purchase decisions, based on reliable cost/worth. This paper has given a simulation approach in modelling and dealing with uncertainties in electrical distribution system reliability evaluation and used exponential distribution for evaluation of reliability of distribution system. It is validated using SOAP UI that the developed MCS service for calculating the reliability indices is platform independent, user friendly and performance oriented

\section{CONCLUSION}

Reliability evaluation of distribution system through Monte Carlo Simulation technique with feature of interoperability for Bus 2 of RBTS system has been introduced and analyzed using SOAP web services. Monte Carlo simulation technique overcomes the uncertainty in input data and prioritizes variability index. The feature of interoperability allows various distribution companies to access this service from anywhere over the network providing a unique solution for distribution reliability evaluation. An effective web service for estimation of distribution system reliability through SOAP messages in XML format for data exchange among distribution companies has been developed. XML is independent of the programming language and hence are readable by any software. This technology is applied on Bus -2 (Feeder 3) of RBTS system. Primary, secondary and cost worth indices are obtained. This service providing a user-friendly interface can be invoked by utilities during power system planning, expansion and operation. The client seeking the reliability indices need internet connectivity and a web browser to access this web service from anywhere. It is proved that data sharing can be achieved independent of the software systems incorporated in the distribution companies. It can be concluded that the developed service-oriented architecture enables 
communication among remote private players in distribution and at the same time evaluates the reliability indices for complex real-time networks.

\section{REFERENCES}

[1] R. Billinton and R. N. Allan, Reliability evaluation of power Systems, $2^{\text {nd }}$ ed. New York, NY, USA: Plenum, 1996.

[2] Roy Billinton and Ronald N. Allan, Reliability evaluation of engineering systems: concepts and techniques, $2^{\text {nd }} \mathrm{ed}$. Springer Science+Buisiness Media New York, 1992.

[3] R. N. Allan, R. Billinton, I. Sjarief, L. Goel, K. S. So, "A Reliability test system for educational purposes - basic distribution system data and results," IEEE Transactions on Power Systems, Vo1.6, No. 2, May 1991.

[4] Roy Billinton and Satish Jonnavithula, "A test system for teaching overall power system reliability assessment," IEEE Transactions on Power Systems, Vol. 1 1, No. 4, November 1996.

[5] Ch.V. S. S. Sailaja, Dr. P.V. N. Prasad, K.V. Ramanamurthy, "Evaluation of reliability indices using FMEA technique," IEEE, February, 2013.

[6] Roy Billinton, and Peng Wang, "Teaching Distribution System Reliability Evaluation Using Monte Carlo Simulation", IEEE Transactions on Power Systems

[7] C. Bhargava and P.S.R Murthy, "Reliability evaluation of radial distribution system using analytical and time sequential techniques", $7^{\text {th }}$ India international conference on Power Electronics, 2016

[8] Habib daryabad, "Investigating the Effect of Demand Side Management on Power System Reliability", Bulletin of Electrical Engineering and Informatics Vol. 4, No. 2, June 2015, pp. 96 102

[9] Venkata Satheesh Babu K, Madhusudan V, and Ganesh V, "Probabilistic Performance Index based Contingency Screening for Composite Power System Reliability Evaluation", International Journal of Electrical and Computer Engineering (IJECE), Vol. 8, No. 5, October 2018, pp. 2661 2670

[10] Ali S. Dalabeeh, Anwar ALMofleh, Abdallah R. Alzyoud, and Hindi T. Ayman. "Economical and Reliable Expansion Alternative of Composite Power System under Restructuring", International Journal of Electrical and Computer Engineering (IJECE) Vol. 8, No. 6, December 2018, pp. 4790 4799

[11] L. Goel and R. Billinton, "Determination of Reliability Worth for Distribution System Planning", IEEE Transactions on Power Delivery, Vol. 9, No. 3, July 1994.

[12] Roy Billinton and Peng Wang, "Distribution System Reliability Cost/Worth Analysis Using Analytical and Sequential Simulation Technique", IEEE Transactions on Power Systems, Vol. 13, No. 4, November 1998.

[13] Huang Xiaoqing, Jiang Hao, and Xia Anbang, "SOA-Based Integration of Electric Utility in Open Electric Market," IEEE, April 2008.

[14] Yin Guisheng and Cui Cao, "Model for Submarine's Power Distribution System Based on Web Services," IEEE, November, 2010.

[15] P. Anbalagan and V. Ramachandran, "An Enhanced Distributed Model for Reliability Evaluation of Power Distribution Systems," International Journal of Computer and Electrical Engineering, Vol. 3, No. 4, August 2011.

[16] S. Leksawat, A. Schmelter, E. Ortjohann, D. Holtschulte, J. Kortenbruck, and D. Morton2, "Implementation of Communication Model and Web Services for Cluster-Based Power System Operation in Smart Grids," IEEE, November, 2015.

[17] E. Ortjohann, P. Wirasanti, S. Leksawat, A. Schmelter, D. Holtschulte, and J. Kortenbruck, "Web-Based Power Flow Analysis Tool for Automated Distribution Network Control," IEEE, June, 2016

[18] Abdul Wahab Mohamed and Ahmed M. Zeki, "Web Services SOAP Optimization Techniques", 4th IEEE International Conference on Engineering Technologies and Applied Sciences (ICETAS), 2017.

[19] http://www.oracle.com/technetwork/articles/java/webapps-1-138794.html

[20] http://www.oracle.com/technetwork/java/index.html

[21] https://www.w3schools.com/xml/xml_services.asp

[22] https://www.w3schools.com/xml/xml_wsdl.asp

[23] https://www.w3schools.com/xml/xml_soap.asp

\section{BIOGRAPHIES OF AUTHORS}

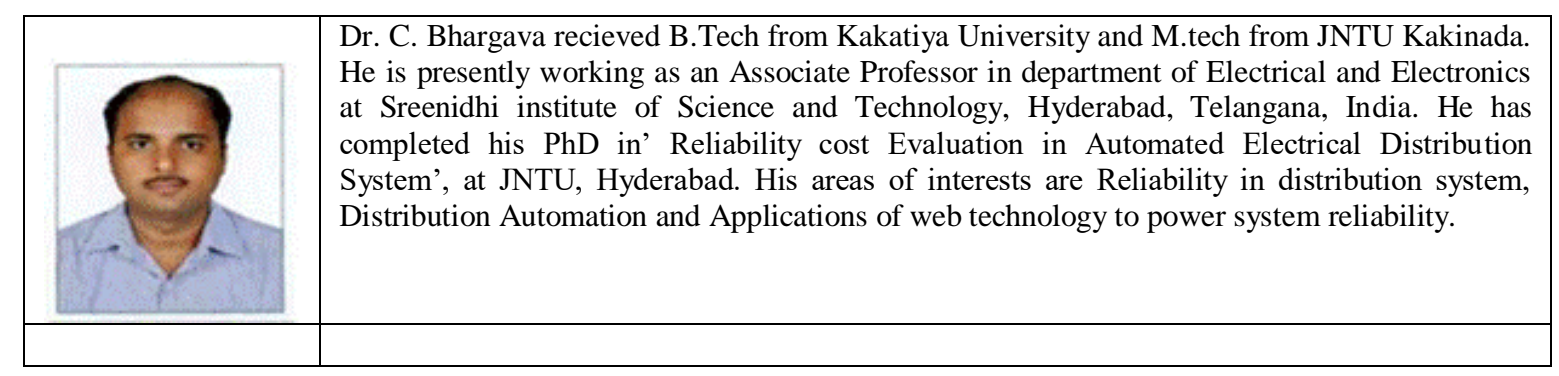

Indonesian J Elec Eng \& Comp Sci, Vol. 14, No. 3, June 2019 : 1065 - 1075 


Renuka Loka received her B.Tech degree in Electrical and Electronics Engineering from Sri
Prakash College of Technology, Rajahmundry, Jawaharlal Nehru Technological University,
Kakinada, India. She is currently pursuing her Masters of Technology inElectrical Power
Engineering from Sreenidhi Institute of Science andTechnology, Jawaharlal Nehru
Technological University, Hyderabad, India. Her research interests include Power System
Reliability analysis, Applications of web technology, Internet of Things to power system
reliability.

